\title{
RÉPARATIONS PAR LES ABEILLES DE BRÈCHES EFFECTUÉES DANS DES CUPULES DE CIRE (1)
}

PAR

\section{VUILLA UME et G. NAULLEAU}

Station de Recherches Apicoles, Bures-sur-Yvette et Faculté des Sciences, Rennes.

Jusqu'à présent nous nous sommes occupés des facteurs agissant sur la construction des cellules royales chez les Abeilles. IARCHEN étudiant la construction des rayons de cire a fait également de nombreuses observations concernant la reconstruction de brèches effectuées dans des rayons et l'obturation de trous percés dans la paroi des cellules (DARCHEN, I958).

Dans un même ordre d'idées nous avons réalisé des brèches dans des cupules (I) rides placées dans des ruches et nous avons observé le comportement des Abeilles dans différents cas.

Nous avons vu par ailleurs (VMILAcMIE) que les Abeilles construisaient les cellules en deux temps:

$I^{\circ}$ Construction de la base des cellules, toujours exécutée en 1'absence d'œufs ou de larves.

$2^{\circ}$ Ce n'est qu'à l'éclosion de la larve que les Abeilles édifieront "le chapean " de la cellule royale.

Nous avons observé également que les Abeilles refusaient d'élever des larves placées dans des cellules présentant un défaut de structure. C'est pour cette raison que nous avons toujours fait les expériences dont il va être question dans des cellules vides.

\section{NATURE DES BRẼCHES ET RÉSULTATS OBTENUS}

\section{I. - Brèches alfectant le bord de la cupule.}

\section{A. - Bord crénelé.} (fig. I).

Après 24 heures de présence dans une ruche, le bord est égalisé par destruction des crêtes. Après remaniement définitif, le bord a l'aspect normal.

(1) Ebauches artificielles en cire re cellules royales d'abeilles.

Annales des Abeilles. - $195 \%$. 
B. - Une brèche affectant la moitié supérieure de la cupule (1). (fig. 2).

Après 24 heures dans les ruches, tout est ramené au même niveau par ablation complète de la paroi laissée intacte. La cupule est alors raccourcie d'une longueur égale à celle de cette paroi ( $a-b$ sur la fig. 2 ).

\section{II. - Brèches afiectant la luase des cellules, le bord restant intact.}

\section{A. -4 ou 8 trous de $1,5 \mathrm{~mm}$ de diamètre dans la moitié supérieure de la cupule.}

(fig. 3 et 3 bis).

Ires trous sont bouchés grossièrement et réunis intérieurement par un début d'opercule qui réduit de près de moitié le diamètre de la cupule. Le colmatage fait toujours saillie à l'intérieur de la cupule ; l'épaisseur de la cire à l'endroit des brèches est inférieure à l'épaisseur de la cupule normale (photos $n^{0}$ I et $n^{0} 2$ ). I es Abeilles ramènent ensuite toute la partie supérieure au niveau de l'opercule (photo $1^{\circ} 2$, cellule de gatuche).

\section{B. -8 trous de $1,5 \mathrm{~mm}$ de diamètre dans la partie inférieure (fig. 4; photo $n^{0} 3$ ).}

Elles réparent grossièrement ces trous et réduisent la partie supérieure de la cellule de quelques millimètres, comme elles le font d'habitude, sans aller cette fois jusqu'aux brèches. L'opercule n'est plus alors construit à l'intérieur de la cellule.

\section{C. - 2 fentes parallèles au bord et opposées entre elles (fig. 5).}

Les fentes larges de $I, 5 \mathrm{~mm}$, à $I, 5 \mathrm{~mm}$ du bord, laissent subsister une couronne de cire à la partie supérieure d'une cupule raccourcie de $3 \mathrm{~mm}$. Cette couronne était rattachée à la cupule par deux bandes latérales de $\mathrm{I}, 5 \mathrm{~mm}$ de largeur. Comme dans le cas des trous dans la partie supérieure de la cupule, les Abeilles, après avoir rebouché grossièrement les fentes et construit à l'intérieur un début d'opercule, détruisent la partie supérieure jusqu'aux brèches (photo $\mathrm{n}^{\circ} 4$ ).

\section{D. - 2 brèches identiques, à la partie inférieure des cupules} (photo $\mathrm{n}^{0} 5$ ).

Comme dans le cas des 8 petits trous placés à la partie inférieure, les Abeilles ne placent pas d'opercule à l'intérieur des cupules, elles raccourcissent seulement le bord de quelques millimètres.

(1) I a partie supérieure de la cupule, par opposition au fond, est la partie voisine du bord. Dans la ruche, louverture est nomalement dirigé vers le bas. La moitié supérieure se trouve alors cn dessous de la partie inférieure. 


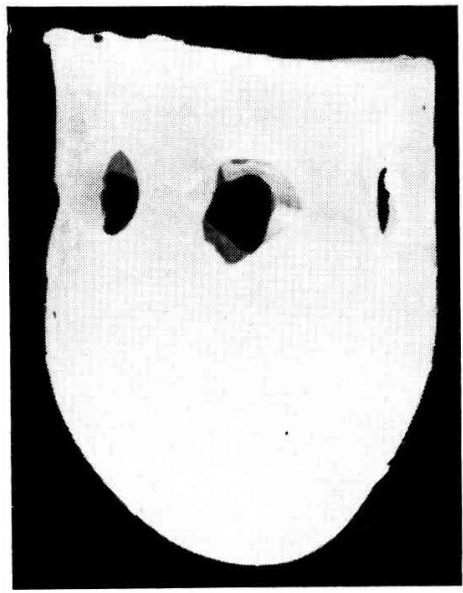

(a)

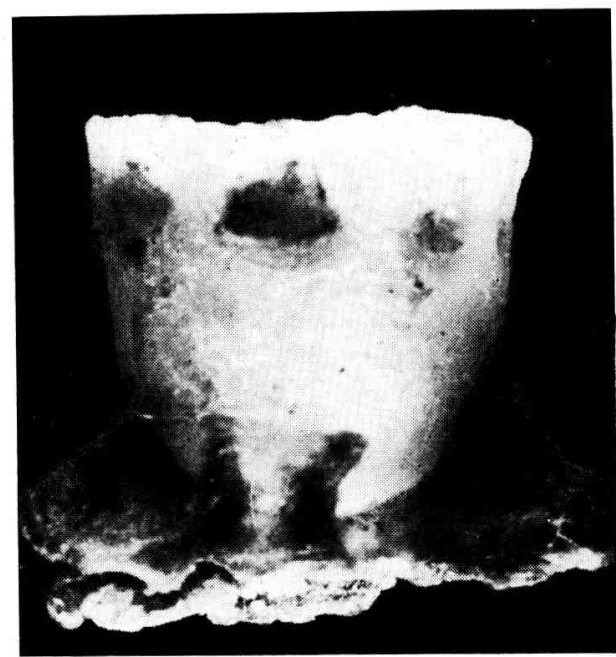

$\left\{\begin{array}{l}2 \\ (2)\end{array}\right.$

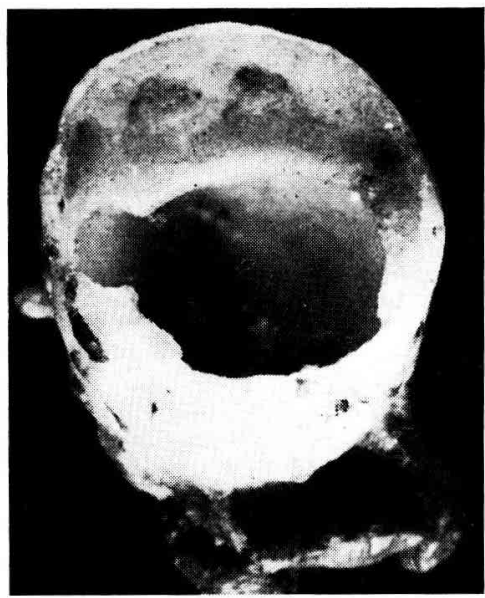

(c)

Pиoto r. -- (a) (upule percée de 8 petits trous daus sa moitie superieure. - (b) Mérré cupule après un court sejour dans lin ruche. Les briches sont grossicrenent houchées. -- (c) Ment chose vue de dessus. Notez les réparations et la presence d'un deni operoule à löntérien de la cupule, au niveat des brerhes. (Photos (ioillot)

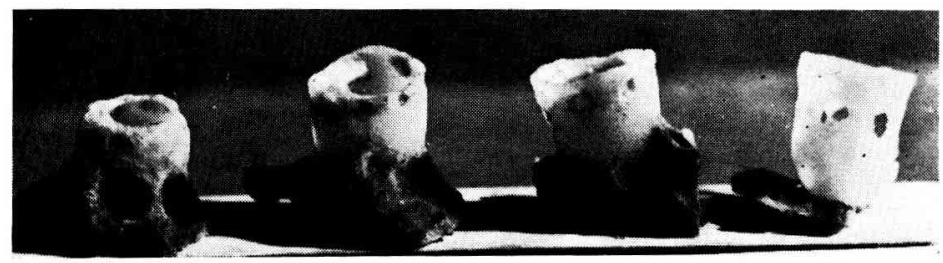

Paroto 2. - - Méme chose que sur lia photo r.

La partie audelit des briches de la cupule de droite a che detruite. 


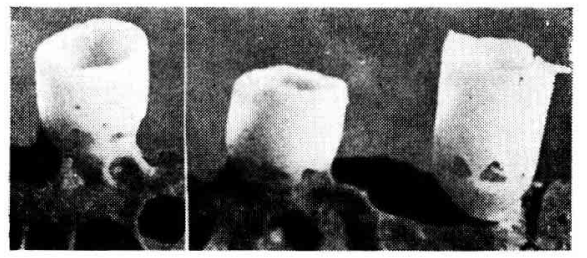

PJumo . - Trous effectués dans la partie inferieure de la rujule.

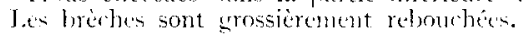

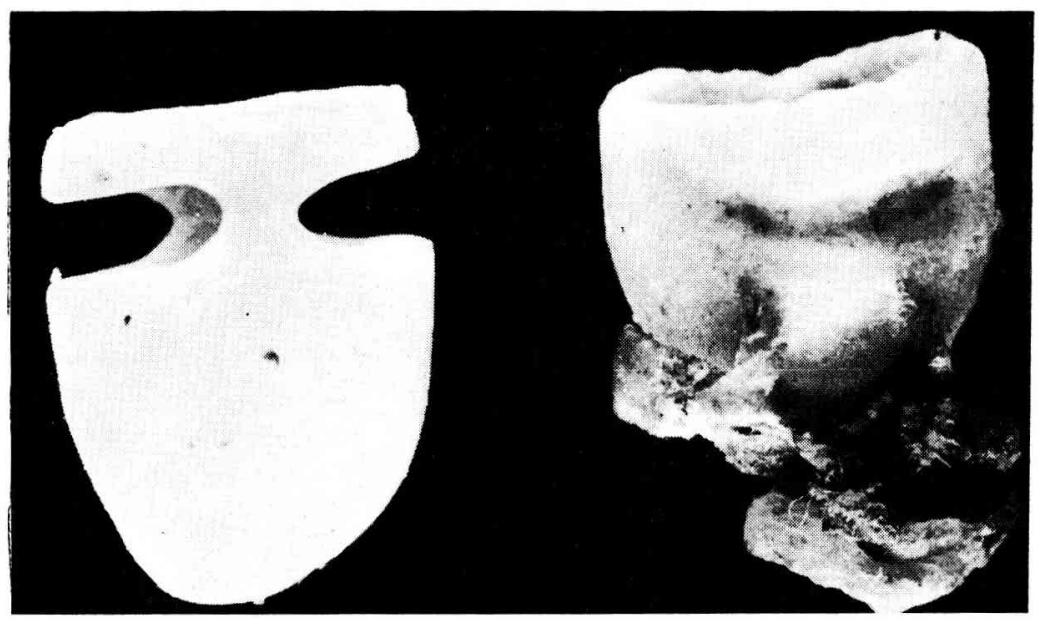

Pnoto \&. - 2 brêches laresé, latérales au borl et situées dans la moitié sujérieure de la cupule. (Photos (ioillot).

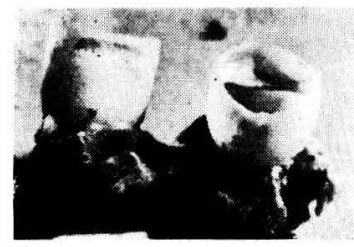

Pното $+\left(^{1}\right)$. - Kemarguer la présence du début dopercule construit à lintérieur de la cupule, al niveau des breches.

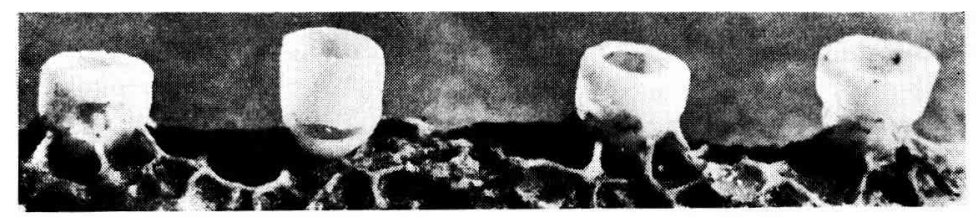

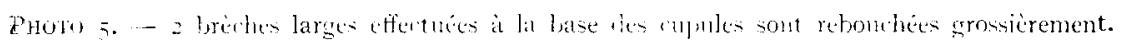




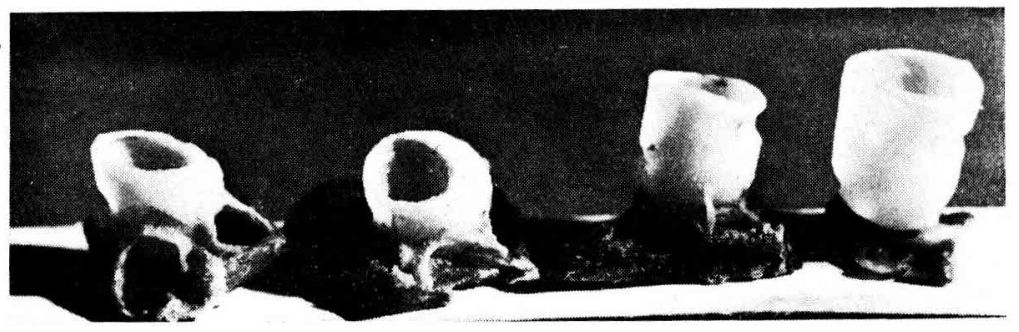

Putron 6. - Noir texte waphere la al Il.

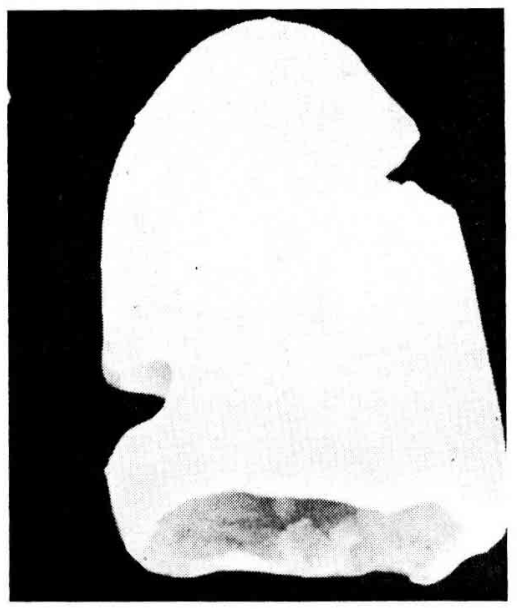

$(t)$

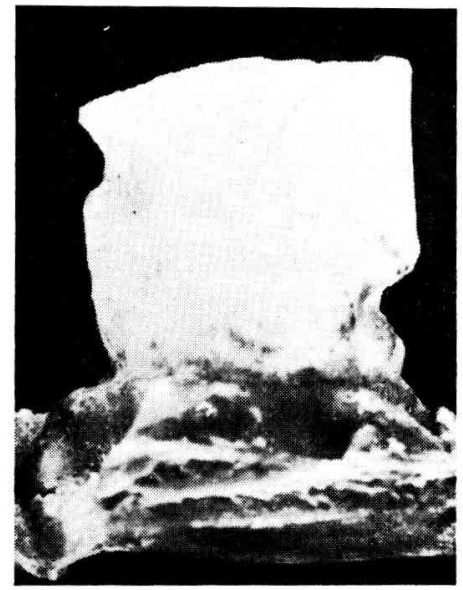

(b)

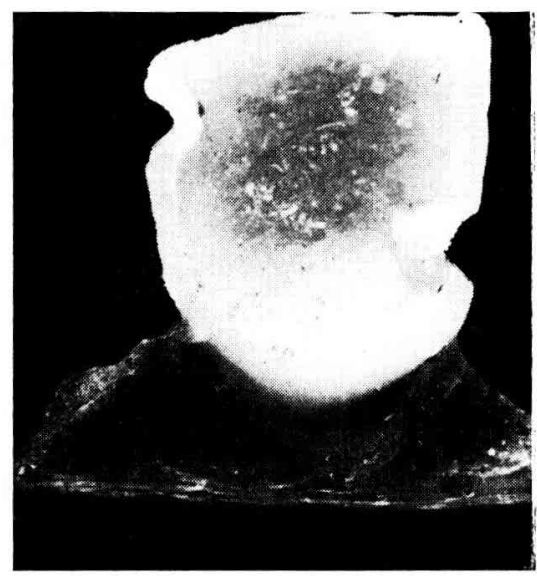

(c) 


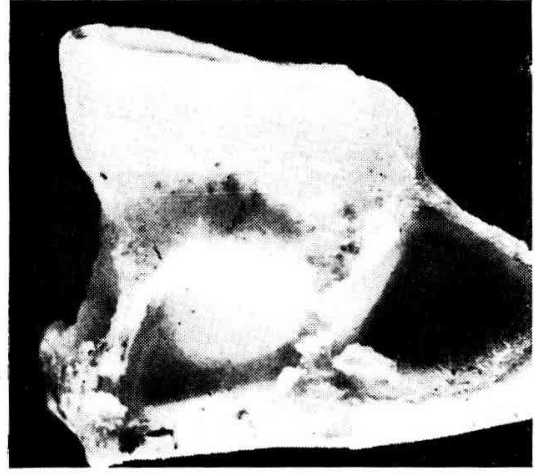

(d)

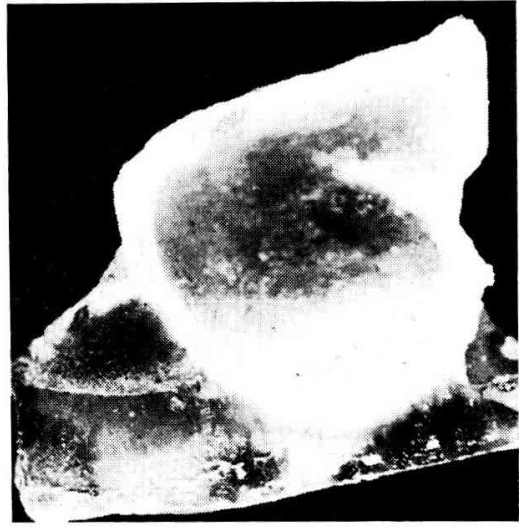

(i)

Puotor bis. - (Voir texte chapitre li de II). - (a) (upule an moment de la mise en ruche. - (b) Même chose a ries reparation grossiere des breches. - $(c)$ id. en couje. Noter la structure de la cupule au nivan des réparations -- (d) Alene howe apres racourcissement all nivean de la breche superivere. … (e) (bulue rle (d). (l'hotos (ionilot)

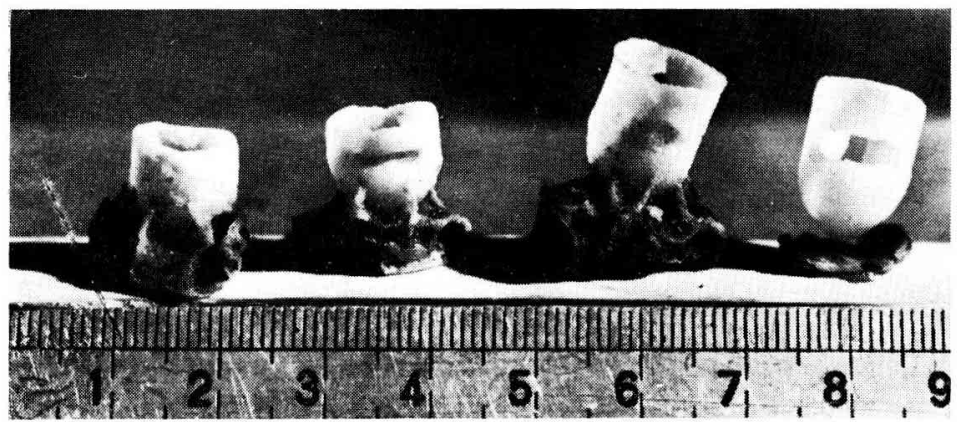

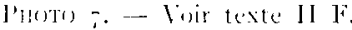

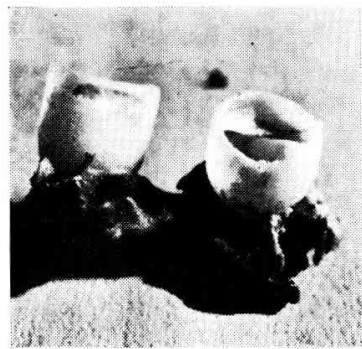

Pung s. - Voir texte ll li sur la cupule de droite. Notez loperenle construit au nivean de la brèche horizontale, et notez la reguration grossiere de la breche verticale. 

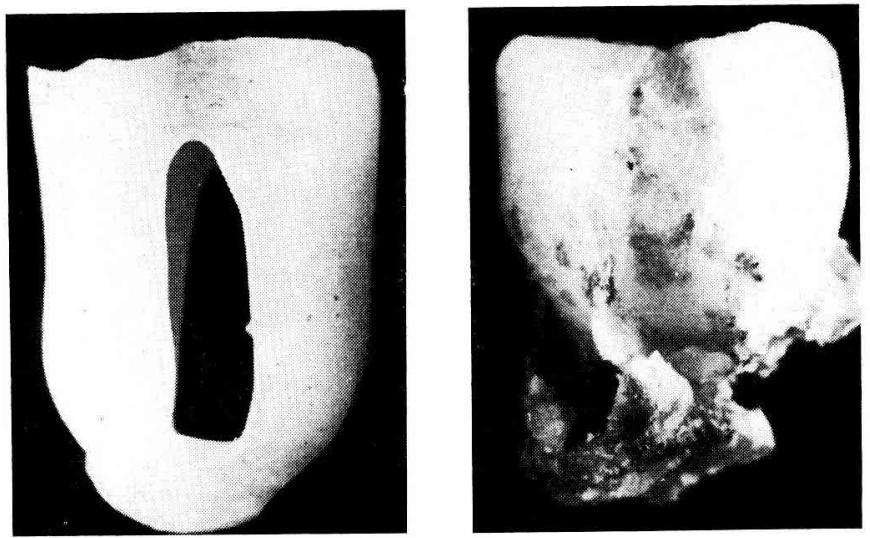

Pното 9 et 9 bis. - Voir texte II G.

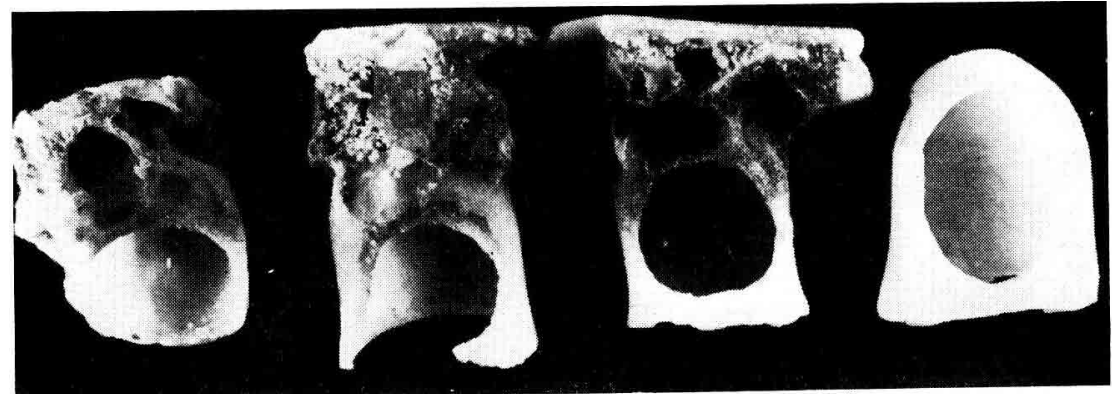

Plioto Io. - Voir texte II II.

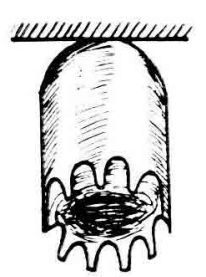

Fig. 1

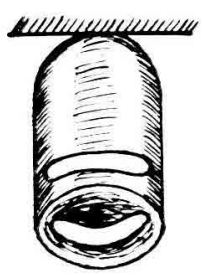

Fig. 5

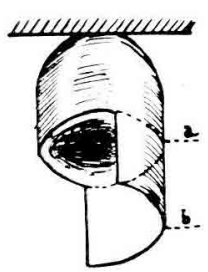

Fig. 2

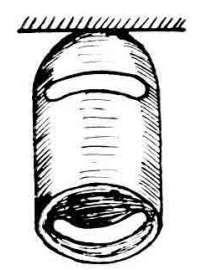

Fig. 6

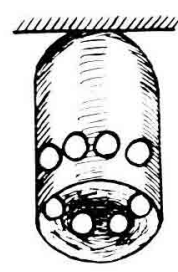

Fig. 3

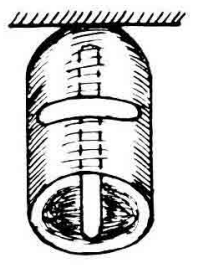

Fig. 7

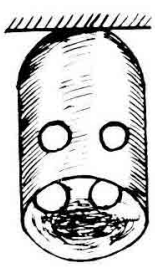

Fig. 3 bis

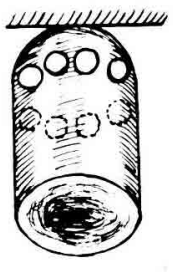

Fig. 4

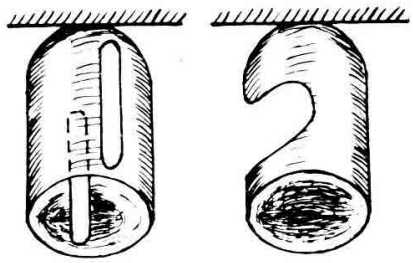

Fig. 8

Fig.9 


\section{E. -2 brèches opposées, l'une à la partie inférieure des cupules, l'autre à la partie supérieure}

(fig. 6).

Elles réparent les 2 brèches, placent un début d'opercule et coupent oblicuement la cupule au niveau de la brèche supérieure (photo 6 ).

\section{F. -2 brèches opposées perpendiculaires}

(fig. 7).

Lilles réparent les 2 brèches, placent un début d'opercule sur la brêche horizontale et coupent an-rlessus de cet opercule (photos $11^{\circ} \mathrm{z}$ et 8 ).

\section{G. - 2 brèches de même grandeur verticales et opposées} (fig. 8).

Elles réparent les brèches et raccourcissent les cupules (photo g).

\section{H. -1 brèche latérale importante}

(fig. 9).

Eilles ne réparent pas la brèche mais compent la cellule au niveau de celle-là (photo $1^{0}$ ro).

\section{CONCLUSIONS}

Nous voyons que la dimension des brêches est capitale; elles sont tonjours obturées si elles sont de petite taille $(5,5 \mathrm{~mm})$ meeme si on les a pratiquées en grand nombre. Inans le cas contraire (grosse brèche latérale) les Abeilles sont obligées cle détruire toute la partie (uui se trouve au-delà de la brèche.

Ia position de la brèche joue égalenent un róle important. Ia partie inférieure de la cupule semble moinss sujette à remanienents que la partie supérieure, puisque les raccourcissements après réparation ne se font que lorsque la brèche affecte la partie supérieure.

Signalons également qu'un opercule partiel n'est construit en face des brèches que dans le cas ou celles-ci affectent la partie supérieure de la cuptle.

Darchins a étudié le conportement des Abeilles vis-à-vis de brèches effectuées non dans des cupules comme nonts lavons fait, mais dans des cellules 11ormales d'ourrières ou de mâles, ou dans des rayons. Il a remarqué que: "les Abeilles bouchent facilement les orifices, même importants, percés clans les fonds ont parois des alvéoles et se servent pour cela de cire vierge récupérée sur les rayons (roir aussi Mfrisir). I,es reconstructions se divisent toujonts en deux temps : a) apport de matérianx informes sur les parties détruites; $b$ ) sculpture de l'ensemble par râclage avec les mandibules". (DARCHEX). 
Nous voyous donc que les résultats qu'a obtenus IARchex avec les cellules normales se rapprochent de cenx que 11ous avons obtenus avec les cupules de cire. Cependant, nous avoms remarqué que l'importance de la brèche joutait un grand rôle; en effet, les brèches sont toujours obturées si elles sont de petite taille, par contre, dans le cas de brèches trop larges elles ne sont pas réparées. Un autre point est également différent : toutes les brèches petites on grandes, faites sur le bord de la cupule, ne sont jamais réparées. Finfin, la réparation est toujours assez grossière et son épaisseur correspond assez rarement à celle de la cupule oi sont effectuées les brèches.

Nous avons vu par ailleurs que la base de la cellule rovale naturelle était construite par les Abeilles dans des conditions bien définies; les stimuli régissant la construction de cette base n'étant pas les mêmes: que céux régissant la construction de la partie supérieure de la cellule rosale. Cette base de la cellule royale a donc pour l'A beille une forme et une structure bien définie. Flle ne supporte en ancun cas un défaut dans sa construction. I'Abeille a donc un comportenent tout autre que les Abeilles maçonnes chez qui le souvenir cinéto-musculaire semble l'emporter sur la structure de la construction. Des brèches effectuées dans le nid ne sont en effet pas réparées (F'HRTON).

I1 arrive cependant que les Abeilles maçonnes réparent les brèches effectuées dans le nid mais cela est fort discuté et dépend de nombreuses conditions en particulier de l'âge des individus et de la saison (TERLAINE).

\section{RIEERIACES BIBLIOGRAPHIOUES}

FERTON (Ch.). -- La vie des abeilles et des guêpes, p. I-376, Ig23.

Darchien (R.). - Constructions sociales chez Apis mellifica; Truc. Ie Intern.

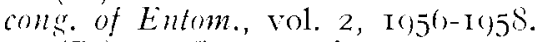

DARCHix (R.). - Constructions et reconstructions de la cellule des rayons d'Apis mellifica. Insectes sociaur, tome $\mathrm{V}, n^{\prime \prime} 4,1958$.

Meyer (W.). - Ueber die Bauarbeiten an den Brut und Honigzellen im Bienen Volk (Apis mellifica L.) (These de doctorat, Berlin).

VERIAINI: (L.). - Instinct and intelligence in Hymenoptera. Journ. Psychol. 29, p. $784-816,665,743$, I032 h.

VeriaINi: (I.). - - Instinct and intelligence in Hymenoptera. Bull. Soc. Ron. Sci. Liège, 2, $24 \hat{s}-253,665,1933$ a.

Vunlitame (II.) - Nonvelles données sur la psychophrsiologie de l'élerage des Reines cliez Apis mellifica. (suite) Amn. Abeille. No2 I I3I3s, I9.5\%. 